\title{
MBTPS2 mutations cause defective regulated intramembrane proteolysis in X-linked osteogenesis imperfecta
}

Uschi Lindert ${ }^{1, \star}$, Wayne A. Cabral ${ }^{2, \star}$, Surasawadee Ausavarat ${ }^{3,4, \star, \dagger}$, Siraprapa Tongkobpetch ${ }^{3,4}$, Katja Ludin ${ }^{5}$, Aileen M. Barnes ${ }^{2}$, Patra Yeetong ${ }^{3,4, \dagger}$, Maryann Weis ${ }^{6}$, Birgit Krabichler ${ }^{7}$, Chalurmpon Srichomthong ${ }^{3,4}$, Elena N. Makareeva ${ }^{8}$, Andreas R. Janecke ${ }^{7,9}$, Sergey Leikin ${ }^{8}$, Benno Röthlisberger ${ }^{5}$, Marianne Rohrbach ${ }^{1}$, Ingo Kennerknecht ${ }^{10}$, David R. Eyre ${ }^{6}$, Kanya Suphapeetiporn ${ }^{3,4, \star}$, Cecilia Giunta ${ }^{1, \star}$, Joan C. Marini ${ }^{2, \star}$ \& Vorasuk Shotelersuk ${ }^{3,4}$

Osteogenesis imperfecta (OI) is a collagen-related bone dysplasia. We identified an X-linked recessive form of $\mathrm{Ol}$ caused by defects in MBTPS2, which encodes site-2 metalloprotease (S2P). MBTPS2 missense mutations in two independent kindreds with moderate/severe OI cause substitutions at highly conserved S2P residues. Mutant S2P has normal stability, but impaired functioning in regulated intramembrane proteolysis (RIP) of OASIS, ATF6 and SREBP transcription factors, consistent with decreased proband secretion of type I collagen. Further, hydroxylation of the collagen lysine residue (K87) critical for crosslinking is reduced in proband bone tissue, consistent with decreased lysyl hydroxylase 1 in proband osteoblasts. Reduced collagen crosslinks presumptively undermine bone strength. Also, proband osteoblasts have broadly defective differentiation. These mutations provide evidence that RIP plays a fundamental role in normal bone development.

\footnotetext{
${ }^{1}$ Division of Metabolism, Connective Tissue Unit and Children's Research Center, University Children's Hospital Zurich, Zurich 8032 , Switzerland. ${ }^{2}$ Section on Heritable Disorders of Bone and Extracellular Matrix, National Institute of Child Health and Human Development, National Institutes of Health, Bethesda, Maryland 20892, USA. ${ }^{3}$ Center of Excellence for Medical Genetics, Department of Pediatrics, Faculty of Medicine, Chulalongkorn University, Bangkok 10330 , Thailand. ${ }^{4}$ Excellence Center for Medical Genetics, King Chulalongkorn Memorial Hospital, The Thai Red Cross Society, Bangkok 10330, Thailand.

${ }^{5}$ Center for Laboratory Medicine, Department of Medical Genetics, Kantonsspital Aarau, Aarau 5001, Switzerland. ${ }^{6}$ Department of Orthopedics and Sports Medicine, University of Washington, Seattle, Washington 98195, USA. ${ }^{7}$ Division of Human Genetics, Medical University of Innsbruck, Innsbruck 6020, Austria. ${ }^{8}$ Section on Physical Biochemistry, National Institute of Child Health and Human Development, National Institutes of Health, Bethesda, Maryland 20892, USA. ${ }^{9}$ Department of Pediatrics I, Medical University of Innsbruck, Innsbruck 6020, Austria. ${ }^{10}$ Institute of Human Genetics, Westfälische Wilhelms University, Münster 48149, Germany. ${ }^{\star}$ These authors contributed equally to this work. $†$ Present addresses: Division of Nuclear Medicine, Department of Radiology, Faculty of Medicine Siriraj Hospital, Mahidol University, Bangkok 10700, Thailand (S.A.); Division of Human Genetics, Department of Botany, Faculty of Science, Chulalongkorn University, Bangkok 10330, Thailand (P.Y.). Correspondence and requests for materials should be addressed to J.C.M. (email: oidoc@helix.nih.gov).
} 
O steogenesis imperfecta (OI), or 'brittle bone disease', is a well-known heritable skeletal dysplasia. Affected individuals have low bone mass and increased fracture susceptibility. OI is genetically heterogeneous. Most cases have autosomal-dominant mutations in the type I collagen genes, COL1A1 or COL1A2 (ref. 1). More recently, multiple genes causing mostly autosomal recessive forms of OI were delineated, generating a new paradigm for OI as a collagen-related disorder. The products of these genes (CRTAP, LEPRE1, PPIB, FKBP10, PLOD2, SERPINH1, BMP1, SERPINF1, IFITM5 and $S P A R C$ ) interact with collagen post-translationally for prolyl 3-hydroxylation, folding, processing, fibrillogenesis, cross-linking and/or mineralization ${ }^{1,2}$. No cases of X-linked OI have been reported.

Regulated intramembrane proteolysis (RIP) involves cleavage of membrane-spanning regulatory proteins by proteases within the plane of the membrane ${ }^{3}$. The three intramembrane cleaving protease (iCLiP) families include serine proteases, site- 2 metalloprotease (S2P) and aspartyl proteases. The best-described RIP-mediated signalling in humans involves sequential cleavage of diverse substrates by site-1 protease (S1P), a serine protease encoded by membrane-bound transcription factor peptidase, site 1 (MBTPS1), and S2P, encoded by membrane-bound transcription factor peptidase, site 2 (MBTPS2). S1P and S2P are located in the Golgi membrane. They cleave regulatory proteins transported from the ER membrane in times of ER stress or decreased sterol metabolites, releasing mature $\mathrm{N}$-terminal fragments that shuttle to the nucleus and activate gene transcription. These substrates include old astrocyte specifically induced substance (OASIS)/cAMP responsive element binding protein 3-like 1 (CREB3L1), a transcription factor expressed in astrocytes and osteoblasts, activating transcription factor 6 (ATF6), a component of the unfolded protein response pathway and sterol regulatory element binding protein (SREBP), involved in cholesterol synthesis ${ }^{4-6}$. MBTPS2 missense mutations were previously identified in the related dermatological conditions IFAP/BRESHECK (ichthyosis follicularis, atrichia, and photophobia, OMIM \#308205) 7,8 and keratosis follicularis spinulosa decalvans (KFSD, X-linked, OMIM \#308800) ${ }^{9}$.

In this report, we present two independent OI pedigrees with an X-linked inheritance pattern, and without symptoms of IFAP/ KFSD. Linkage analysis and next-generation sequencing (NGS) identified a novel MBTPS2 missense mutation in each pedigree. The resulting substitutions are in or near the S2P NPDG motif crucial for metal ion coordination ${ }^{10}$. Mutant S2P protein is stable but cleavage or activation of S2P substrates OASIS and ATF6, respectively, is impaired, consistent with reduced proband collagen secretion. Osteoblasts with mutant S2P have defective differentiation, and bone tissue collagen from one proband has decreased hydroxylation of the critical crosslinking lysine residue. These X-linked OI-causing mutations extend RIP functioning to normal bone formation.

\section{Results}

Clinical features. Family I. From an extended Thai OI pedigree, 6 of 12 affected males were examined (Fig. 1a,b and Supplementary Table 1). The proband (P1/I; V7) had prenatal fractures of ribs and long bones (Fig. 2a). At 2 years, his physical exam was notable for moderate short stature, blue sclerae, pectus carinatum and bowing of lower extremity long bones. His L2-L4 bone mineral density $z$-score $=-4.7\left(0.315 \mathrm{~g} \mathrm{~cm}^{-2}\right)$. Affected adult males had fractures beginning during gestation, short stature, white sclerae, variable scoliosis and pectal deformity, striking tibial anterior angulation and generalized osteopenia (see Case Reports).
Family II. A non-consanguineous German pedigree has two affected males, the 26-year-old proband (P1/II) and his 68-year-old maternal uncle (P2/II) (Fig. 2c; Supplementary Fig. 2a and Supplementary Table 1). As a newborn, the proband was noted to have bowing of humeri, radii and tibiae, and fractures of femora, ribs and clavicles, as well as generalized osteopenia (Fig. 2b and Supplementary Fig. 2b). Both patients had numerous fractures of upper and lower extremity long bones during childhood, but fractures declined post puberty. Both have white sclerae, kyphoscoliosis with anterior vertebral wedging and short stature, with final height of 5-6-year-old males. The uncle has severe pectus excavatum.

None of the affected individuals had ichthyosis follicularis, alopecia, photophobia, intellectual disability or seizures.

Mutation identification. Sequencing of a set of genes involved in OI and bone fragility (ALPL, BMP1, COL1A1, COL1A2, CREB3L1, CRTAP, FKBP10, IFITM5, LEPRE1, LRP5, PLOD2, PLS3, PPIB, SERPINF1, SERPINH1, SP7, TMEM38B and WNT1) did not reveal functional changes in the probands of either pedigree. Each proband had a neutral COL1 single-nucleotide polymorphism (SNP) that was inherited from his healthy father (Supplementary Fig. 3). Interestingly, the urinary lysyl pyridinoline (LP) to hydroxylysyl pyridinoline (HP) ratio, which has been suggested as a diagnostic marker for $\mathrm{OI}^{11}$, was markedly elevated in an adult from Family I and two adults from Family II (0.305-0.450 patients versus controls $0.211 \pm 0.008)$ and in the index case from Family I (0.319 versus paediatric controls $0.208 \pm 0.030$ ) (Supplementary Table 2). The LP/HP ratio was not elevated in a carrier from each OI Family, or in a case of IFAP. The elevated LP/HP ratio is similar to findings in a mouse model of type IX $\mathrm{OI}^{12}$, although X-OI probands have normal PPIB sequences.

X-chromosome inactivation studies of five obligate carriers from Family I showed a skewed pattern of inactivation (Supplementary Fig. 4). Linkage analysis of the X-chromosome in Family I defined a $21.6-\mathrm{Mb}$ critical region on Xp22 from markers DXS7108 to DXS1067 (LOD score 3.31) (Fig. 1b and Supplementary Table 3). Comparative genomic hybridization (CGH) analysis did not reveal imbalances in the critical region, which was then sequenced by NGS in affected male IV17 and unaffected male IV10. After excluding known SNPs, and variants present in the unaffected relative, two candidates remained. A mutation in MBTPS2 exon 11, c.1376A $>\mathrm{G}$ (nucleotide: NM_015884.3, GRCh38) predicting a p.N459S substitution (protein: NP_056968.1), was confirmed by Sanger sequencing of proband DNA (Fig. 2c), segregated with OI in the pedigree (Fig. 1b) and was absent in $644 \mathrm{X}$ chromosomes of unrelated Thai controls (181 females, 282 males). A FAM48B1 variant, c.532G $>$ A (p.V178M), occurred in 4 of 22 alleles from Thai controls (Supplementary Table 4).

In Family II, SNP-genome-wide linkage analysis supported $\mathrm{X}$-linked recessive inheritance, despite normal $\mathrm{X}$-inactivation (0.70) in the mother of P1/II, with a positive LOD score in a $30-\mathrm{Mb}$ region of chromosome $\mathrm{X}$ between rs11094708 and rs5906168 (Supplementary Fig. 1a and Supplementary Table 5). In view of the modest positive LOD score, the entire X-exome was sequenced by NGS, resulting in two variants with population frequencies below $0.5 \%$ within the $30-\mathrm{Mb}$ critical region. The first variant, a hemizygous c.1778G $>$ A (p.R593H) missense SNP in CCDC120, was detected in proband P1/II but not in his affected uncle (P2/II). The second variant, a hemizygous c.1515G $>\mathrm{C}$ mutation in MBTPS2 (p.L505F; Supplementary Fig. 1b), co-segregated with the phenotype and was also found in a heterozygous state in the proband's mother (Fig. $2 \mathrm{~d}$ ). 


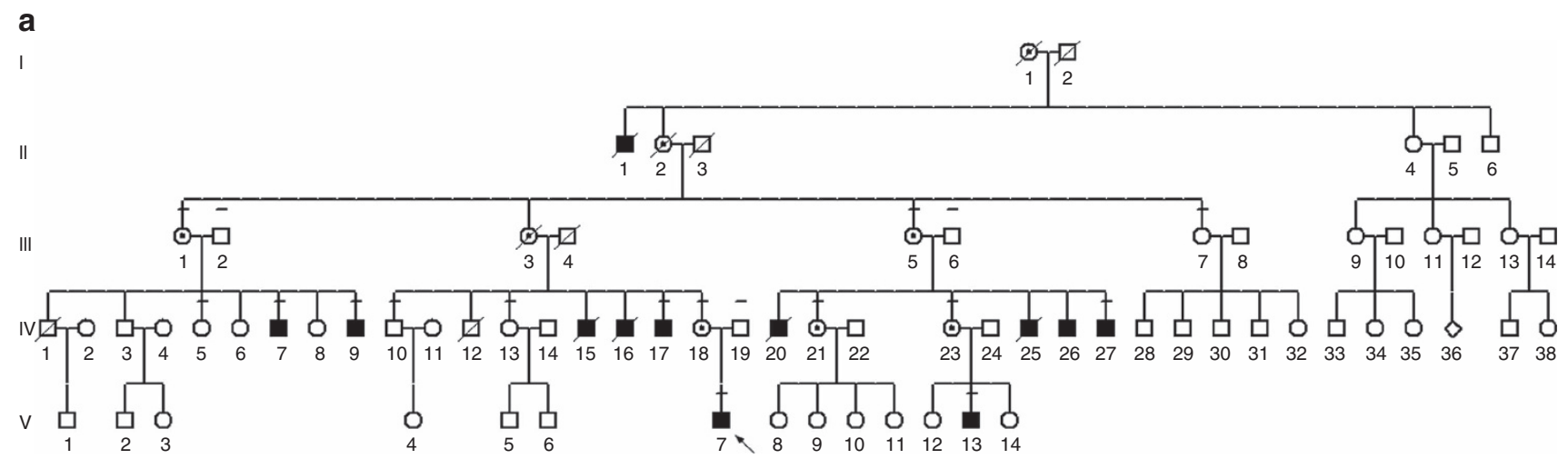

b

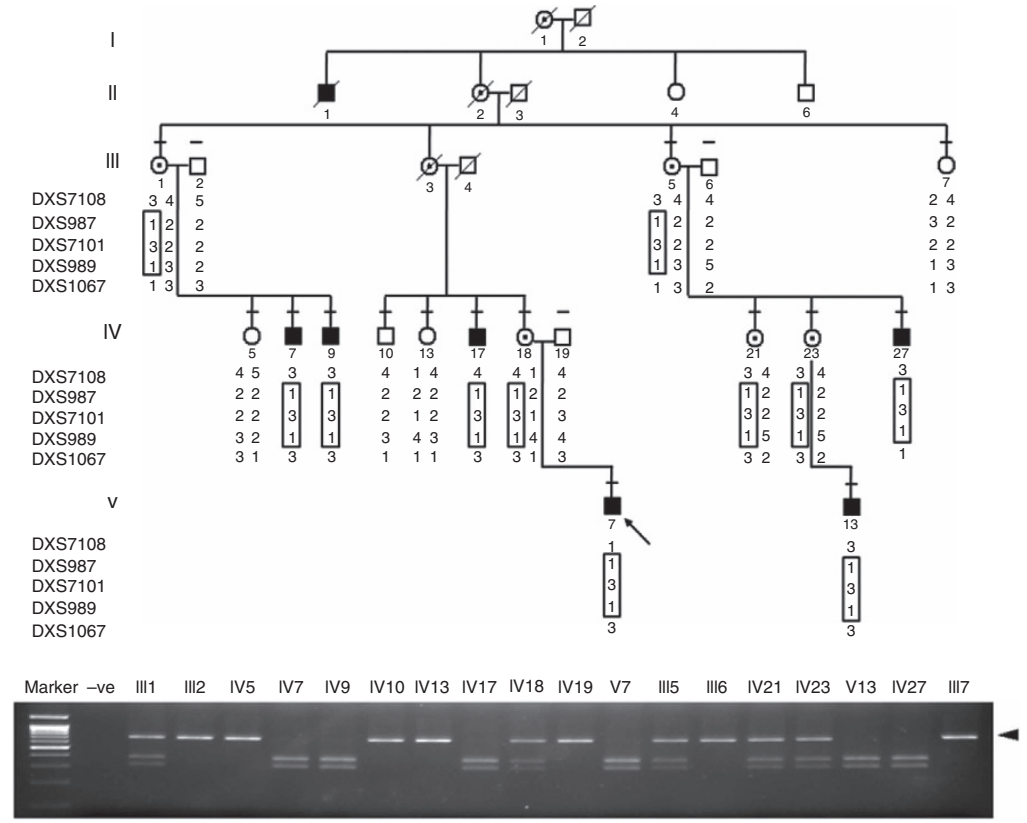

C

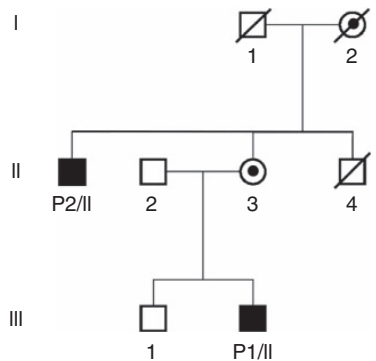

Figure 1 | Extended pedigree of Family I with linkage and segregation analyses. (a) Extended pedigree of Family I. The pedigree contains 12 affected members (blackened symbols), with seven living and five deceased (diagonally crossed). Clinical assessments and blood samples were obtained from 18 individuals (short horizontal bars above symbols), including six affected. Dotted circles denote obligate carriers. Arrow indicates Proband P1/I.

(b) Linkage and segregation analysis. Thai pedigree shows linkage of mutation with $\mathrm{X}$ chromosome and cosegregation of the mutation with phenotype. X-chromosomal markers are shown from Xpter to Xcen (full list of markers in Supplementary Table 3). Linked markers in squares are shown in relation to the members' status. The MBTPS2 c.1376A > G (p.N459S) mutation segregated with phenotype in all affected members. The mutation introduces a Bsgl cleavage site, resulting in 375 and 305 bp bands. The normal allele generates the undigested 680 bp band. Marker $=100$ bp marker; - ve, negative control with no DNA added; an arrowhead indicates the $680 \mathrm{bp}$ band. (c) Pedigree of Family IIA. The segregation analysis was done on all family members still alive at the time of sampling, that is all but individuals $\mathrm{I} / 1, \mathrm{I} / 2$ and $\mathrm{II} / 4$. The index patient $\mathrm{P} 1 / \mathrm{II}$ and his maternal uncle P2/II show very similar clinical findings compatible with the diagnosis osteogenesis imperfecta type III/IV. The mother of P1/II is of normal height, whereas the maternal grandmother is only $155 \mathrm{~cm}(<3 r d$ centile), but does not show any signs of osteogenesis imperfecta.

Multiple programs (Polyphen2, SIFT, Provean, MutationTaster) predict both MBTPS2 (S2P) variants p.N459S and p.L505F as pathogenic since they are likely to interfere with function (Supplementary Table 6). Residues N459 and L505 are highly conserved in S2P, as identified by ClustalX (Fig. 2e), as well as ClustalW and Boxshade (for p.L505F). Neither of the MBTPS2 mutations, c.1376A $>$ G (p.N459S) and c.1515G $>$ C (p.L505F) are present in the Leiden Open Variation Database (LOVD), Exome Aggregation Consortium (ExAC), the Exome Variant Server (EVS), 1000 Genomes Browser and dbSNP Build 144 variant databases (Supplementary Table 6). N459 is the first residue of the NPDG motif, which is important for metal ion coordination ${ }^{10}$.

Effect of MBTPS2 mutations on transcripts and protein. MBTPS2 transcript levels were not reduced in fibroblasts with OI,
IFAP (p.R429H) or KFSD (p.N508S) mutations, by real-time RT-PCR (Fig. 3a). In addition, S2P protein displayed normal stability in these cells on immunoblot (Fig. 3b).

Effect of mutant S2P on RIP functions. We examined the impact of S2P substitutions on activation of RIP substrates OASIS, ATF6 and SREBP (Fig. 4a-c). On western blot, mature intracellular OASIS cleavage fragment $(50 \mathrm{kDa}, \mathrm{S} 1 \mathrm{P} / \mathrm{S} 2 \mathrm{P}$ cleaved $)$ was decreased, while the $55-\mathrm{kDa}$ cleavage product (S1P cleaved) was increased, in fibroblasts with each mutation and in S2P p.L505F osteoblasts, indicating S2P cleavage impairment in OI probands comparable to IFAP and KFSD cells (Fig. 4a). Treatment with ALLN ( $N$-acetyl-leucyl-leucyl-norleucinal) to prevent degradation of OASIS cleavage products increased the total OASIS detected in all fibroblast and osteoblast cell lines, as well as the relative proportion of fully cleaved OASIS in normal 
control, IFAP and KFSD fibroblasts and normal control osteoblasts, more than in OI cells (Fig. 4a). Treatment with tunicamycin to induce ER stress had no further effect on OASIS cleavage.
An ATF6 reporter was co-transfected with MBTPS2 expression constructs containing normal, OI-causing (p.N459S or p.L505F) or IFAP/BRESHECK (p.R429H) ${ }^{7,8}$ sequences into CHO-M19 a
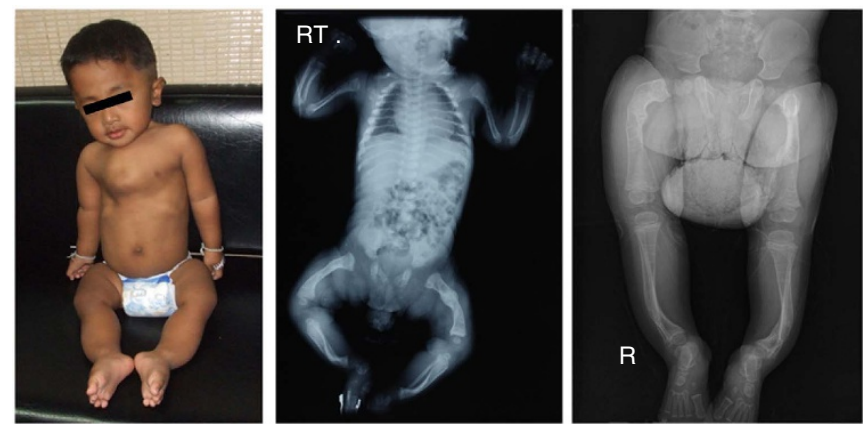

b

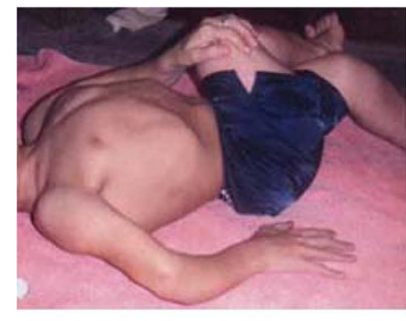

C

Father

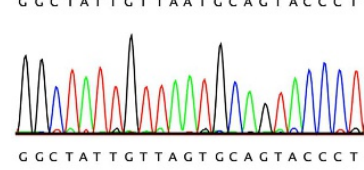

$\mathrm{P} 1 / \mathrm{l}$ (N459S)

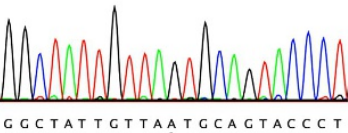

Mother
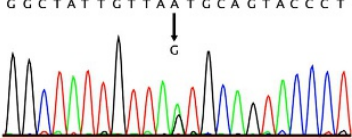

d
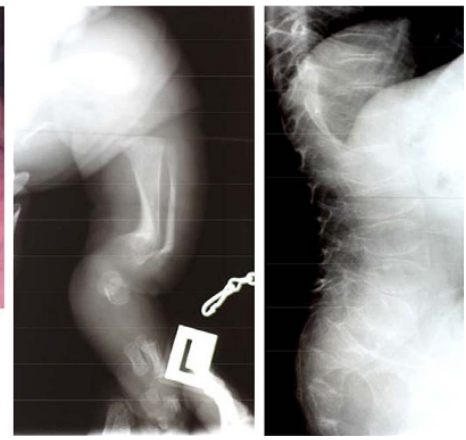

Father
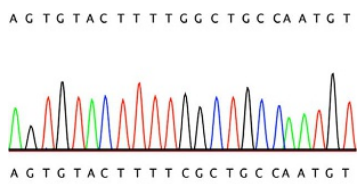

$\mathrm{P} 1 / \mathrm{ll}(\mathrm{L} 505 \mathrm{~F})$

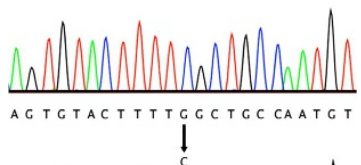

Mother

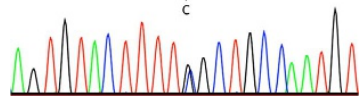

e

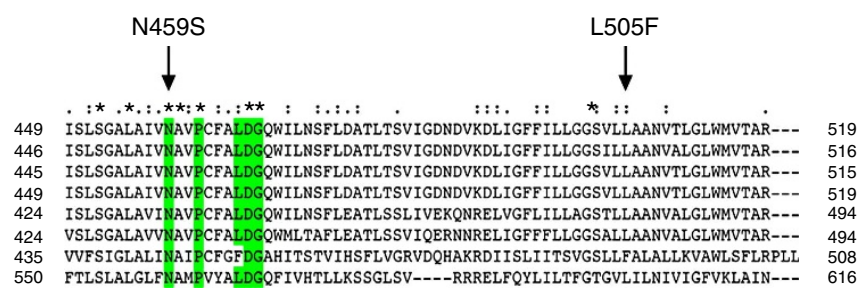

Homo sapiens

Bos Taurus

Mus musculus

Rattus norvegicus

Gallus gallus

Drosophila melanogaster

Caenorhabditis elegans

Figure 2 | Clinical features of probands and mutation identification. (a) Left, Proband 1/I had blue sclerae, pectus carinatum and anteriorly bowed legs at 2 years of age. Centre, radiographs showed fractures of ribs, humeri, femora and tibiae on day 1 of life. Right, undertubulation and minimal cortex of lower long bones at 2 years of age. (b) Left, Proband 1/II at age 20 years presenting with significant rhizomelia of upper and lower extremities. Centre, radiographs at birth showed bowed tibiae and fibulae (left leg shown). Right, radiographs revealed kyphoscoliosis with anterior vertebral wedging, and flat, biconcave vertebral bodies with significant osteoporosis at 13 years of age. (c) MBTPS2 genomic DNA sequence reveals the c.1376A $>G$ mutation (p.N459S) in proband P1/I and his heterozygous mother, but not in the proband's father. (d) Sequence of P1/II genomic DNA shows the MBTPS2 c.1515G > C mutation (p.L505F), which also occurs in heterozygous form in his mother, but is not present in the proband's father. (e) Species comparison of the S2P amino-acid sequence according to ClustalX. Residues shaded in green denote the NPDG motif required for enzymatic active site metal ion coordination.

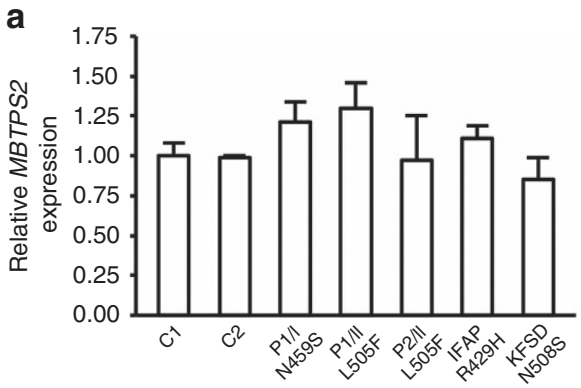

b

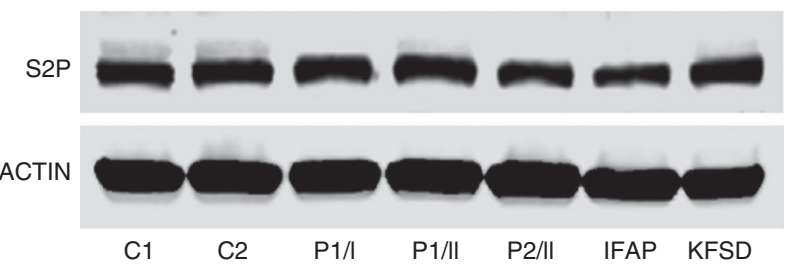

Figure 3 | Expression of MBTPS2 in OI and IFAP fibroblasts. (a) Quantification of MBTPS2 transcripts by real-time RT-PCR shows no significant differences. (b) S2P protein levels are normal in OI, IFAP and KFSD fibroblasts, as detected by western blot. No significant differences in MBTPS2 expression were detected between control and proband fibroblasts. Error bars, s.d. 
a
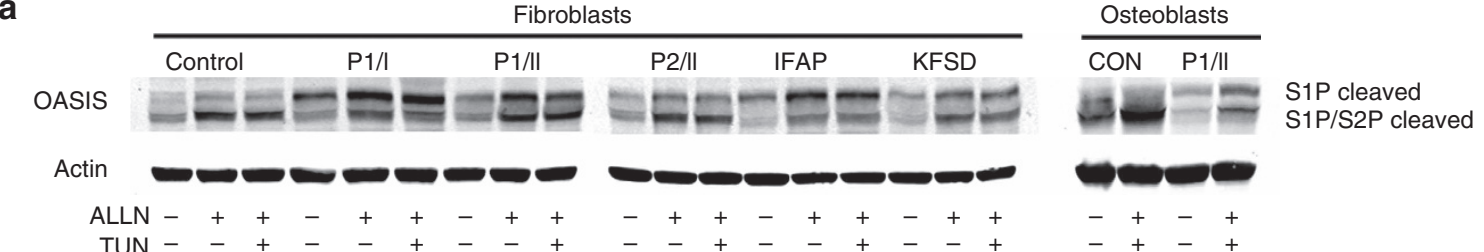

b
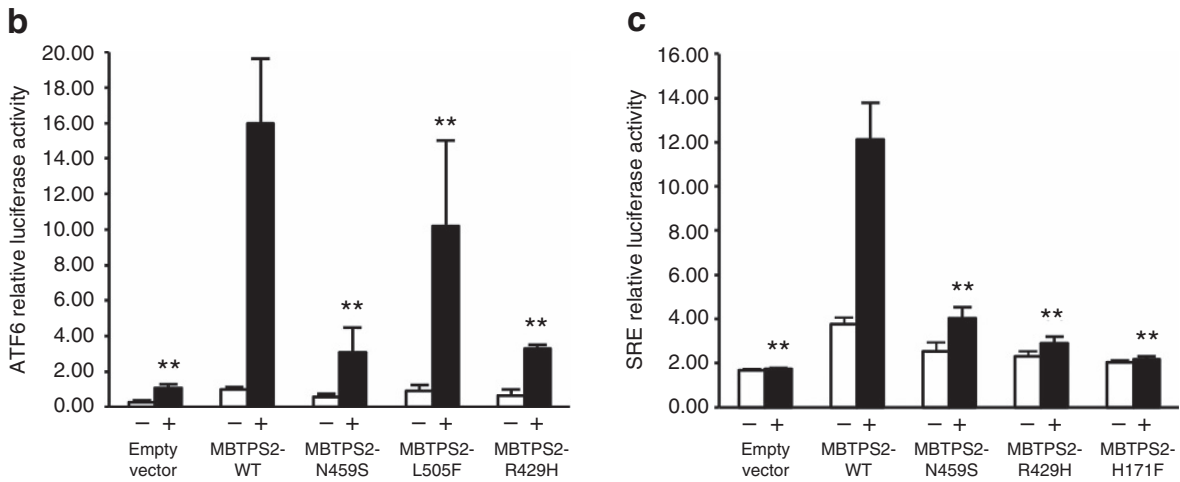

Figure 4 | Functional studies of the MBTPS2 variants. (a) Western blots of OASIS cleavage by S1P and S2P. Control fibroblast and osteoblast lysates show cleavage of OASIS to its mature active form (S1P/S2P cleaved). Cells from OI, IFAP and KFSD probands contain increased partially cleaved OASIS (S1P cleaved) due to impaired cleavage by S2P (TUN, tunicamycin; ALLN, N-[N-(N-acetyl-L-leucyl)-L-leucyl]-L-norleucine). (b) Luciferase assays of MBTPS2 activation of ATF6 reporters upon induction of stress (+). Renilla luciferase was used to normalize the transfection efficiency. WT, N459S, R429H, and L505F indicate that cells were transfected with normal or mutant MBTPS2 constructs. ${ }^{\star \star} P$, wild-type versus empty vector, $\mathrm{N} 459 \mathrm{~S}$, R429H, and L505F $<0.001$ by ANOVA. (c) Luciferase assays of MBTPS2 activation of SRE reporters on induction of stress by depletion of sterols ( + ). Renilla luciferase was used to normalize the transfection efficiency. The results were represented as mean \pm s.d. WT, N459S, R429H and H171F indicate that cells were transfected with normal or mutant MBTPS2 constructs. ${ }^{\star \star}$, wild-type versus empty vector, N459S, R429H and H171F $<0.001$ by ANOVA. Error bars, s.d.

cells lacking MBTPS2 (Fig. 4b). The transcriptional activation of ATF6 after tunicamycin-induced ER stress was significantly decreased in CHO-M19 cells expressing mutant constructs (p.N459S, p.L505F, p.R429H) versus normal MBTPS2 $(P<0.01)$ (Fig. $4 \mathrm{~b}$ and Supplementary Table 7 ), as measured by luciferase activity. Interestingly, the depression of ATF6 activation from p.L505F transfection was less marked than with p.N459S or IFAP-causing p.R429H. This is similar to the degree of impaired OASIS cleavage in untreated mutant fibroblasts, in which p.N459S and IFAP cells had relatively more uncleaved OASIS than did p.L505F cells, although all mutants did significantly impair cleavage versus control.

Studies of SRE reporter co-transfection with MBTPS2 expression constructs were done with only p.N459S and IFAP/KFSD mutants (Fig. 4c, Supplementary Table 8). Luciferase activation was suppressed in all cells expressing mutant constructs in sterol-free media.

Effect of defective S2P on type I collagen biochemistry. Since OI is a collagen-related bone dysplasia and fully processed OASIS is reported to activate Colla1 transcription ${ }^{13}$, we examined type I collagen biochemistry in fibroblasts and osteoblasts with mutant S2P. Collagen transcripts were variable in mutant cells, with decreased COL1A1 transcripts detected in fibroblasts of OI proband 1/I (p.N459S), IFAP (p.R429H) and KFSD (p.N508S) fibroblasts, but not in OI fibroblasts or osteoblasts with the S2P p.L505F mutation (Fig. 5a).

Fibroblasts from OI probands secreted a significantly reduced amount of type I collagen (20-73\% of control), as did IFAP and KFSD fibroblasts $(\approx 40 \%)$ (Fig. $5 \mathrm{~b}$ ). Proband osteoblasts were insufficient for this assay. Electrophoretic migration of proband collagen (Fig. 5c) was normal, consistent with normal thermal stability $\left(T_{\mathrm{m}}\right)$ and helical resistance to proteolytic digestion at physiologic temperature (Supplementary Fig. 5a,b). Collagen deposited into matrix in culture by S2P p.N459S fibroblasts from P1/I had a decreased proportion of collagen containing mature cross-links ( $10 \%$ versus $22 \%$ of collagen deposited by normal control fibroblasts) (Supplementary Fig. 5c), suggesting that hydroxylation of the type I collagen K87 residue crucial to crosslinking may be impaired.

Effect of mutant S2P on bone tissue and cells. Tissue and osteoblast characteristics of OI were demonstrated in a bone sample from P1/II (p.L505F). Osteoblasts differentiated in culture revealed impaired expression of transcripts related to osteoblast maturation and RIP pathways (Fig. 6). Alkaline phosphatase $(A L P L)$ expression is about half of control in mid- to late differentiation. OASIS (CREB3L1) expression was significantly reduced throughout osteoblast differentiation. Mature OASIS normally complexes with SMAD4 to upregulate expression of matrix genes ${ }^{14}$. However, SMAD4 transcripts were reduced throughout p.L505F osteoblast differentiation, underlying the reduced expression of the proteoglycan matrilin-1 (ref. 15). Interestingly, COL1A1 expression in P1/II osteoblasts was comparable to control (Fig. 5a).

Collagen extracted directly from P1/II bone tissue had less than half the normal level of hydroxylation of the lysine (K87) critical for collagen crosslinking in both collagen alpha chains (43\% versus $90 \%$ control in $\alpha 1(\mathrm{I})$, and $7 \%$ versus $43 \%$ control in $\alpha 2(\mathrm{I}))$ (Fig. 7a and Supplementary Fig. 6). This is consistent with both the proband's increased urinary $\mathrm{LP} / \mathrm{HP}$ ratio and reduced crosslinked collagen deposited into matrix. However, hydroxylation of collagen carboxyl-terminal crosslink residues K930/933 was normal in each chain (Fig. 7a and Supplementary Fig. 6). The enzyme responsible for modification of the crosslinking lysine (K87), lysyl hydroxylase 1 (LH1, encoded by PLOD1), was substantially reduced in S2P p.L505F osteoblast lysates, although the level of CyPB/PPIB, a foldase critical to LH1 function, was 


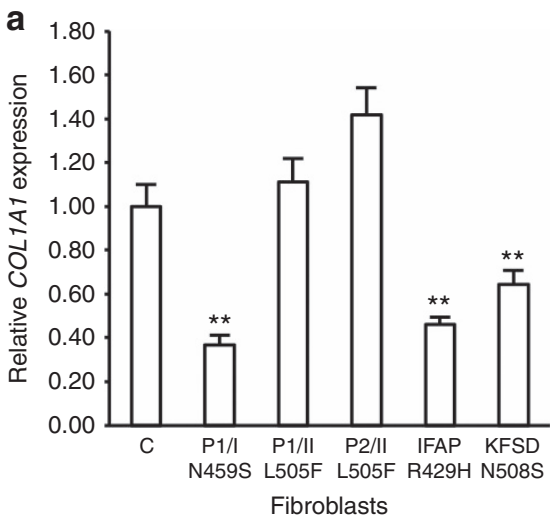

C

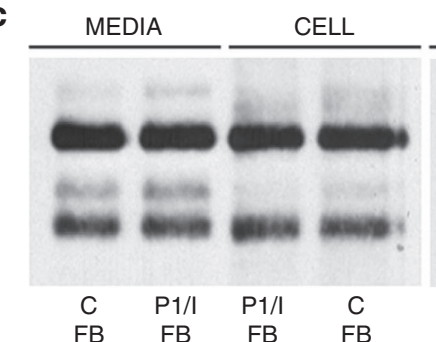

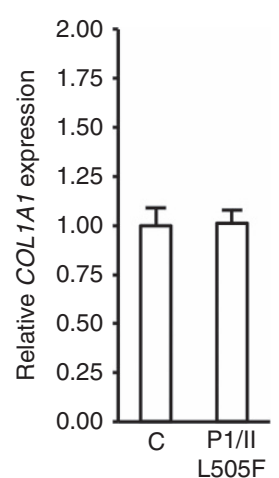

Osteoblasts

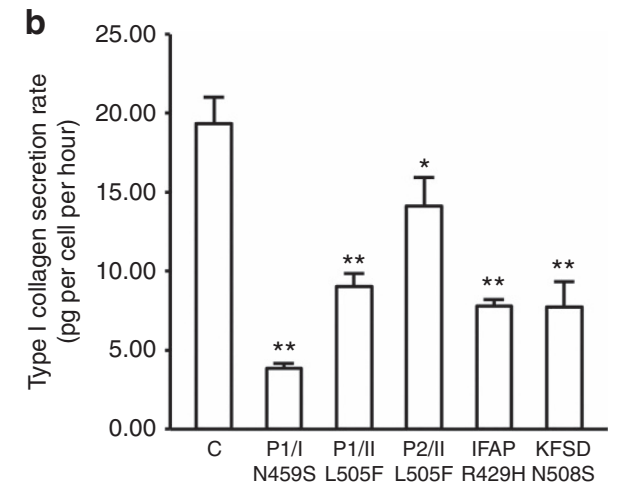

CELL

Figure 5 | Effects of MBTPS2 mutations on type I collagen. (a) Expression of COL1A1 in normal control (C), OI proband, IFAP and KFSD fibroblasts, and $\mathrm{OI}$ and control (C) osteoblasts. (b) Total procollagen secretion by fibroblast lines harbouring MBTPS2 mutations is decreased compared with normal control (C) fibroblasts. (c) Steady-state type I collagen analysis. The electrophoretic migration of collagen alpha chains synthesized by OI proband fibroblasts (FB) and osteoblasts (OB) was equivalent to normal control (C) fibroblast collagen. ${ }^{\star} P<0.05$, ${ }^{\star \star} P<0.001$ by $t$-test. Error bars, s.d.

a

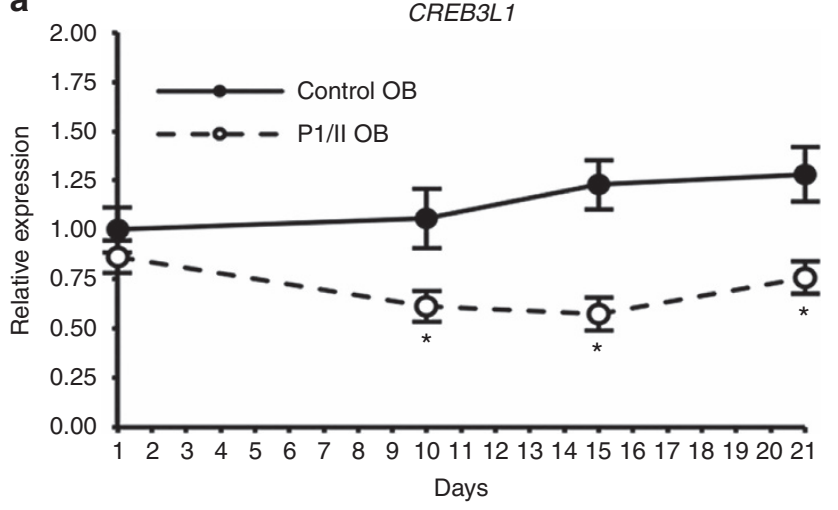

C

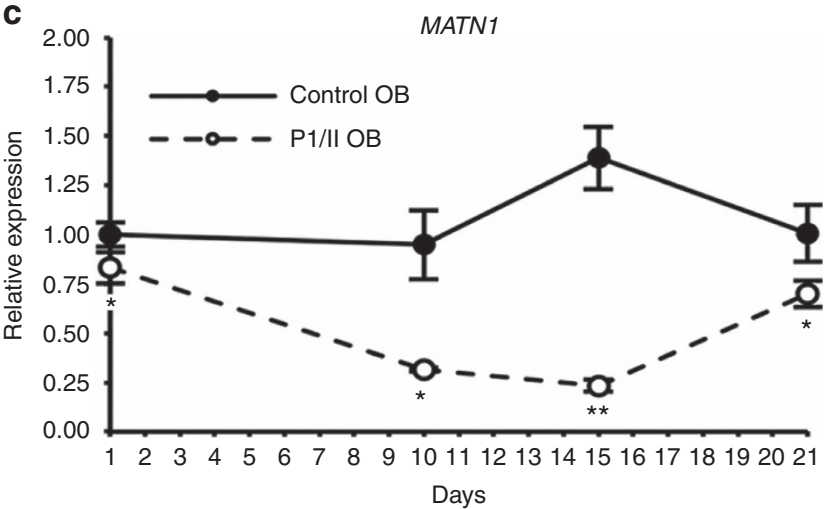

b

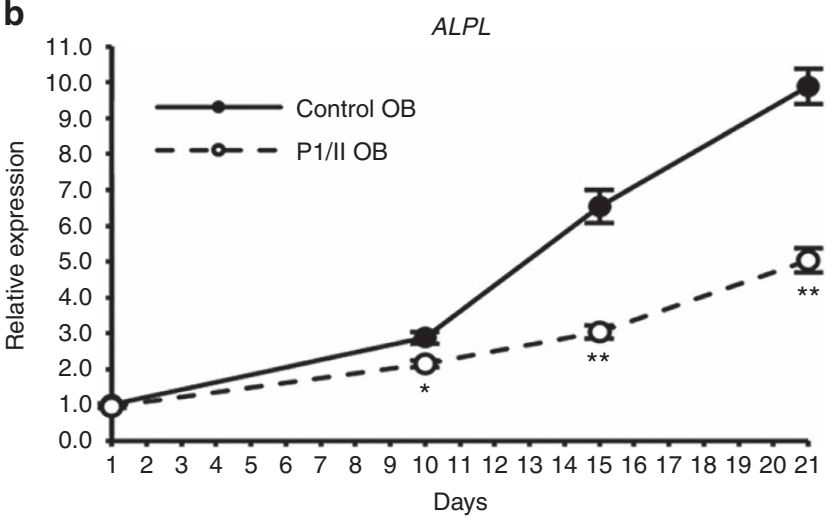

d

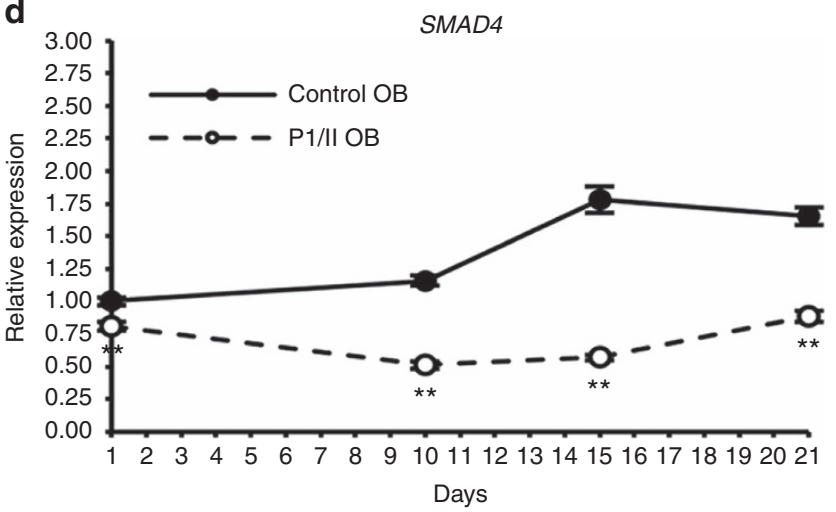

Figure 6 | Effect of S2P deficiency on osteoblast differentiation. (a) Transcripts encoding OASIS (CREB3L1), (b) bone-specific alkaline phosphatase $(A L P L)$, (c) matrilin-1 (MATN1) and (d) Co-SMAD (SMAD4) are decreased in differentiating P1/II versus normal control osteoblasts in culture. ${ }^{\star} P<0.05,{ }^{\star \star} P<0.001$ by $t$-test. Error bars, s.d. 
a
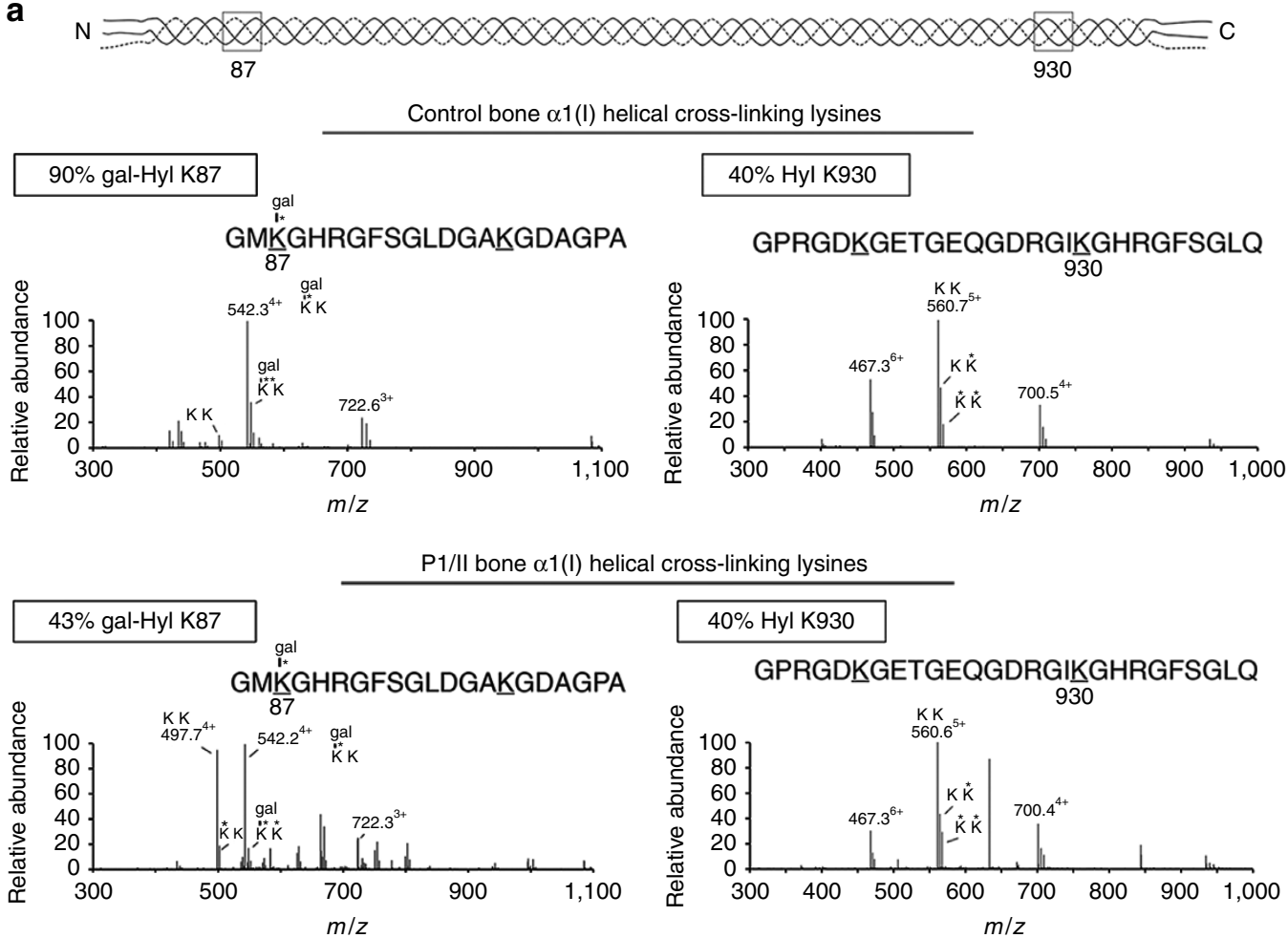

b
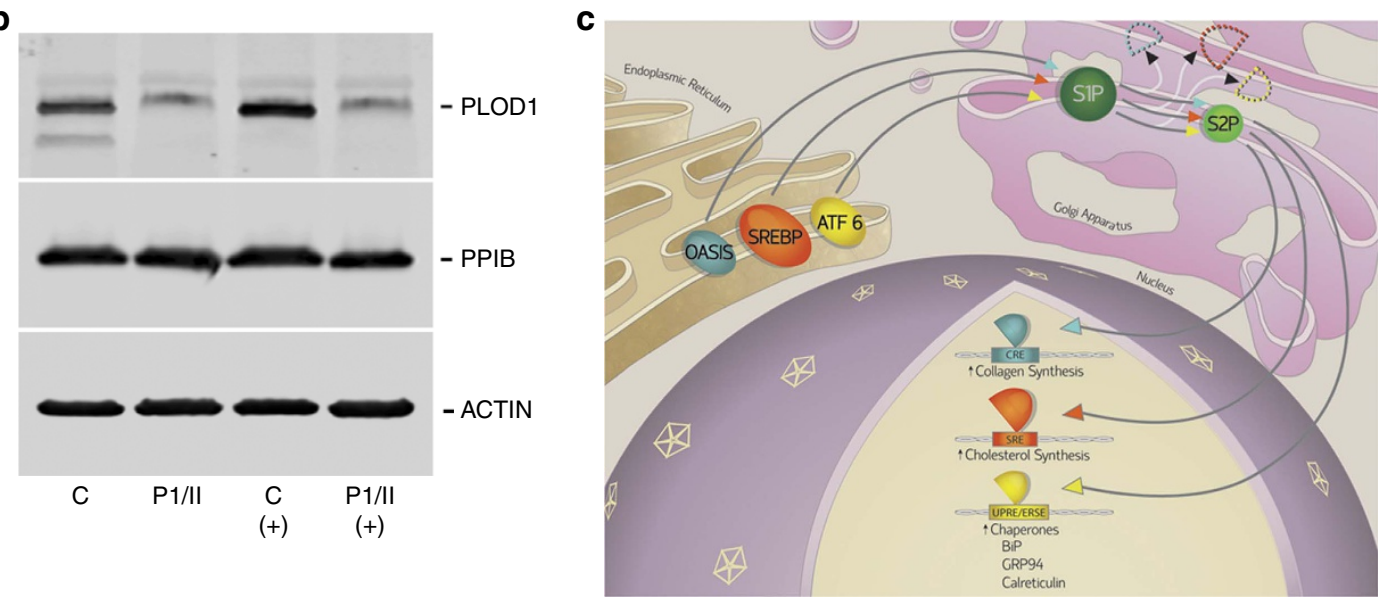

Figure 7 | S2P deficiency causes abnormal post-translational modification of type I collagen. (a) Mass spectrometric analysis of bacterial collagenasedigested peptides of control and P1/II bone type I collagen. Hydroxylation and consequent glycosylation of $\alpha 1(\mathrm{I}) \mathrm{K} 87$ residues, involved in crosslink formation, is decreased in the proband sample by more than one-half, while hydroxylation of $\alpha 1(\mathrm{I}) \mathrm{K} 930$ residues is normal, compared with normal control bone. K, lysine; $\mathrm{K}^{\star}$, hydroxylysine; gal, galactosyl. (b) Immunoblots of lysates from control (C) and P1/II osteoblasts cultured in the absence or presence $(+)$ of tunicamycin, an inhibitor of N-linked glycosylation that induces ER stress and OASIS cleavage. Despite normal levels of CyPB/PPIB, proband cells have significantly decreased LH1/PLOD1 protein, consistent with decreased hydroxylation of collagen lysyl residues (K87) involved in crosslink formation in extracellular matrix. (c) Schematic of RIP-mediated signalling. OASIS, SREBP and ATF6 translocate to the Golgi in response to ER stress or cholesterol depletion, where they are sequentially cleaved by S1P and S2P. The fully processed forms shuttle to the nucleus to activate transcription of genes involved in protein folding, lipid synthesis and extracellular matrix development ${ }^{3,5,13,21}$.

normal (Fig. 7b) ${ }^{12,16}$. PLOD1/LH1 transcripts and protein were not consistently reduced in OI or IFAP/KFSD fibroblasts (Supplementary Fig. 7).

\section{Discussion}

We identified an X-linked form of osteogenesis imperfecta in two independent pedigrees. Phenotypic inheritance pattern, linkage analysis and NGS were used to localize the causative gene in each family to MBTPS2 at Xp22. The gene defects co-segregate with the phenotype in each pedigree and all affected individuals are the sons of obligate carriers. This gene identification extends the inheritance pattern of OI to include X-linked recessive. Notably, the OI phenotype of these MBTPS2 defects is distinct from that of PLS3 mutations, which cause an X-linked form of osteoporosis that is mostly apparent in middle-aged adults, but does cause fractures in some children ${ }^{17}$. Unlike MBTPS2 defects reported here which cause moderate to severe OI, PLS3 defects do not cause a generalized bone dysplasia, changes in bone shape or structure, or secondary features of OI. 
MBTPS2 encodes S2P, an integral membrane protein critical to RIP and essential for cholesterol metabolism ${ }^{18}$. Mutations previously reported to cause substitutions in S2P (Supplementary Fig. 8) underlie a spectrum of dermatological conditions: IFAP syndrome, characterized by a triad of ichthyosis follicularis, atrichia and photophobia ${ }^{7}, \mathrm{KFSD}^{9}$, BRESHECK $^{2}$ sydrome ${ }^{8}$ and Olmsted syndrome ${ }^{19}$. The affected individuals in our OI pedigrees do not have any symptoms associated with these conditions, even into adulthood. Both OI-causing substitutions in S2P are located in highly conserved intramembrane residues near the carboxyl end of the protein, and likely contribute to a specialized function. In Family I, the MBTPS2 missense mutation results in a p.N459S substitution at the first residue of the S2P NPDG motif, which coordinates the zinc metal ion critical to protease catalytic function ${ }^{10}$. In addition, the serine substitution at this site introduces a potential O-GlcNAc glycosylation site (http://www.cbs.dtu.dk/services/YinOYang/), and addition of a bulky group might interfere with $\mathrm{S} 2 \mathrm{P}$ interaction with its substrates. The Family II MBTPS2 mutation results in S2P p.L505F. This substitution, in which leucine is replaced with the bulkier phenylalanine, is predicted to be pathogenic by multiple mutation analysis programs and may also interfere with S2P substrate interactions. Interestingly, the nearby p.N508S and p.N508T have a mild IFAP and KFSD phenotype ${ }^{9}$, and most IFAP mutations are located adjacent to this critical region (p.R429-N508) ${ }^{20}$.

S2P and S1P span the Golgi membrane and function sequentially in RIP to process crucial regulatory substrates transported from the ER membrane in times of ER-stress or sterol restriction, including OASIS, ATF6 and SREBP (Fig. 7c) 3,5,13,21. The mature processed proteins then enter the nucleus where they activate pathways involved in cholesterol homoeostasis, unfolded protein response and bone formation. Our demonstration of S2P functioning in human bone development is supported by prior studies on defects of RIP components in animal models. Both S1P and S2P knockdown zebrafish showed abnormal cartilage development, independent of lipid defects ${ }^{22}$. Cartilage-specific S1P knockout mice (S1Pcko) have a lethal chondrodystrophy, with loss of hypertrophic chondrocytes and absent endochondral bone formation ${ }^{23}$. $\mathrm{S}_{1} \mathrm{P}^{c k o}$ chondrocytes also display differential downregulation of transcripts related to cholesterol and lipid biosynthesis ${ }^{24}$. In addition, ablation of the RIP substrate OASIS, a bZIP transcription factor, causes spontaneous fractures and severe osteopenia in mice ${ }^{13}$ and homozygous deletion of CREB3L1 (OASIS) causes recessive OI in one family ${ }^{25}$.

The MBTPS2 mutations causing X-linked OI do not impair MBTPS2 expression or destabilize S2P protein, nor do MBTPS2 mutations causing IFAP/KFSD. Instead, they disrupt S2P processing of RIP substrates, interfering with their downstream functions. First, both p.N459S and p.L505F fibroblasts and p.L505F osteoblasts display impaired OASIS cleavage. OASIS/CREB3L1 expression is decreased in p.L505F osteoblasts, as is the expression of its nuclear binding target SMAD4 (ref. 14), which, along with the reduced expression of osteoblast maturation marker alkaline phosphatase, indicates a broad defect in osteoblast differentiation. SMAD4 in turn co-activates transcription of matrix proteins such as matrilin-1 (ref. 15), consistent with decreased expression of MATN1 in OI proband P1/II's osteoblasts. Second, type I collagen secretion is decreased in fibroblasts of all OI probands. The combination of normal collagen expression and reduced collagen secretion found in mutant MBTPS2 fibroblasts differs from the expected decreased SMAD4 transcriptional activation of Colla1 (ref. 14), as well as from the murine Oasis knockout, in which reduced Colla1 expression was detected ${ }^{13}$. However, reduced collagen secretion was not detected in fibroblasts of patients with mutant CREB3L1 $(\mathrm{OASIS})^{25}$. Furthermore, S1P ${ }^{\text {cko }}$ chondrocytes retain type IIB procollagen due to a trafficking defect but do not exhibit decreased Col2a1 expression ${ }^{24}$. Finally, the activation of RIP substrates ATF6 and SREBP during ER stress and sterol-free conditions, respectively, were significantly reduced in CHO-M19 cells transfected with reporter constructs containing mutant versus normal MBTPS2.

Notably, bone tissue from one X-OI proband revealed an underlying collagen-related defect. Type I collagen extracted directly from P1/II bone had significantly reduced hydroxylation of helical lysine residue 87 (K87) in both alpha chains, consistent with the proband's increased urinary LP/HP ratio. Although bone was not available from $\mathrm{P} 1 / \mathrm{I}$, his elevated urinary $\mathrm{LP} / \mathrm{HP}$ ratio suggests a similar effect on collagen crosslinking occurs in his bone, while the LP/HP ratio was normal in two X-OI carriers and IFAP/KFSD patients (Supplementary Table 2). K87 is critical for collagen crosslinking in bone, which is a major contributor to bone strength ${ }^{26}$. Similarly reduced hydroxylation of type I collagen K87 to about half of wild-type control values occurs in a Ppib/CyPB knockout mouse model of type IX OI ${ }^{12}$. In the Ppib knockout mouse, the level of LH1/PLOD1, the enzyme which modifies K87, is normal, but its function is impaired by absence of its PPIase, CyPB. In contrast, LH1 protein is reduced in osteoblasts from X-linked OI proband P1/II by an unknown mechanism, although the level of $\mathrm{CyPB}$ is normal. Thus, the bone collagen defect in $\mathrm{X}$-linked OI combines reduced collagen secretion into matrix and impaired collagen crosslinking. Disturbance of additional OASIS functions may contribute to the severe skeletal phenotype of X-linked OI, including apoptosis, downregulation of osteocalcin, osteopontin and bone sialoprotein, and ER stress, which overlaps ATF6 activation ${ }^{27}$.

It is apparent that mutations at different positions in MBTPS2 cause distinct syndromes, with no evidence at this time to suggest a spectrum disorder. A variety of skeletal malformations have been reported in IFAP-family syndromes, specifically vertebral malformation and cleft hands ${ }^{28}$. For example, the p.R429H IFAP mutation studied in our reporter assays causes vertebral abnormalities $^{7,8}$. Short stature, inguinal hernia and microcephaly have also been noted in some IFAP patients. However, IFAP patients do not have the fracture susceptibility or generalized bone dysplasia that are the hallmarks of OI. However, the mechanism by which different mutations in MBTPS2 cause totally different developmental disorders remains unclear. The biochemical studies comparing X-linked OI and IFAP fibroblasts show no clear distinction between phenotypes in cleavage of RIP substrates OASIS, ATF6 and SREBP. The major distinction between syndromes resides in the urinary crosslinks, with elevated $\mathrm{LP} / \mathrm{HP}$ ratios in $\mathrm{X}-\mathrm{OI}$ probands and not in $\mathrm{X}-\mathrm{OI}$ carriers or an IFAP patient. This presents a reasonable hypothesis that impaired hydroxylation and crosslinking of bone collagen is the critical distinction for X-OI versus IFAP. Future studies elucidating the RIP pathways involved in bone development may reveal tissue-specific differences in collagen post-translational modification and crosslinking, or critical S2P bone-related substrates other than OASIS, ATF6 and SREBP. These studies of RIP pathways in bone development are of fundamental importance and will be facilitated by animal models and bone samples from additional patients with both X-OI and IFAP.

\section{Methods}

Detailed case reports. Family I. The proband (P1/I; V7) of Family I, the Thai pedigree, was delivered at 39 weeks gestation by caesarean section due to multiple long bone fractures detected at 4 months gestation. His birth weight was $2,860 \mathrm{gm}$ (10th centile), length $45 \mathrm{~cm}$ ( $<3 \mathrm{rd}$ centile), head circumference $34 \mathrm{~cm}$ (40th centile) and anterior fontanelle $5 \times 5 \mathrm{~cm}$. He had fractures of ribs, humeri, femora and tibiae (Fig. 2a). The proband received pamidronate starting at age 3 weeks. 
He stood with support at 18 months. No additional fractures occurred through 24 months of age, when his weight was $10 \mathrm{~kg}$ (3rd centile), length $76.5 \mathrm{~cm}$ (50th centile for a 13-month-old boy) and head circumference $47.8 \mathrm{~cm}$ (40th centile). He had closed anterior fontanelle, positional plagiocephaly, blue sclerae, normal teeth, pectus carinatum, straight spine and upper extremity long bones but bowing of femora and tibiae. Karyotype showed normal 46, XY.

The pedigree contains 12 affected members, with seven living (Fig. 1a). Clinical assessments and blood samples were obtained from 18 individuals, including six affected (Supplementary Table 1). The proband's 32-year-old mother was healthy with normal weight, height and bone mineral density. The proband and his mother had normal serum calcium, phosphorus, alkaline phosphatase and lipid profiles. Affected adult males had fractures beginning during gestation, white sclerae, normal teeth, head circumference $52-58 \mathrm{~cm}$ (within mean \pm 2.5 s.d. for Thai adults), variable scoliosis and pectal deformity, bowing of extremities with striking anterior angulation of tibiae, generalized osteopenia and final adult stature from 110 to $140 \mathrm{~cm}$ (50th centile for age 5-11 years).

Family II. Patient P1/II is a 26-year-old man (Fig. 2b and Supplementary Fig. 2a). The pregnancy was uneventful and he was delivered spontaneously at term. Birth weight was $2,970 \mathrm{~g}$ (10th-25th centile), with a length of $46 \mathrm{~cm}$ (3rd-10th centile). Direct postpartal bowing of the distal humeri and the proximal radii were indicative of osteogenesis imperfecta. Radiography showed bilateral fractures of the clavicles, several rib fractures and a consolidated prenatal fracture of the left femur (Supplementary Fig. 2b). Between age 14 months and 16 years, numerous fractures occured after only minor and moderate traumatic events, involving the femur 11 times (this stopped after the insertion of Bailey-Dubow elongation rods at age 8 years), the humerus 7 times, the lower arm 2 times and the tibia 3 times. Two fractures of the mandibulae occured after adequate traumatic events. There were no new fractures until age 24 years when one traumatic fracture of the lower leg occured. Treatment with $9 \mathrm{mg} \mathrm{kg}^{-1}$ per day intravenous bisphosphonate was started at age 13 for 3 years. Current height at the age of 26 years is $110 \mathrm{~cm}$ (50th centile for 6-year-old male) and weight is $37 \mathrm{~kg}$. Moderate kyphoscoliosis of the thoracic and lumbar spine is present. There are no signs of dentinogenesis imperfecta and the sclerae are white. An active wheelchair is mainly used. Unsupported standing is possible for a longer time; unsupported walking only a few steps, with support 5-10 m, and on a treadmill more than $1 \mathrm{~km}$.

The maternal uncle $\mathrm{P} 2 / \mathrm{II}$ is a 68 -year-old man who was diagnosed with osteogenesis imperfecta quite soon after birth. Fracture rate and bending of the long bones were similar to P1/II. Since puberty, fractures are rarely observed unless through adequate trauma. He has a severe connatal pectus excavatum. His current height is $105 \mathrm{~cm}$ (50th centile for 5-year-old male) and his weight is $45 \mathrm{~kg}$. Kyphoscoliosis of the thoracic and lumbar spine is more pronounced than in his nephew, and is rather progressive. He has no signs of dentinogenesis imperfecta and his sclerae are white. He can walk slowly with two crutches.

Molecular genetic investigations of Family I. DNA from blood and dermal fibroblasts. Proband and family whole blood and skin samples were collected after written informed consent was obtained under a protocol approved by the Institutional Review Board of the Faculty of Medicine, Chulalongkorn University. High-molecular-weight DNA was extracted from leukocytes of all the available family members, and from cultured skin fibroblasts of the proband, using the ArchivePure DNA Blood Kit (5 PRIME Inc., Gaithersburg, MD), according to the manufacturer's instructions.

$X$-inactivation assay. Eight female members (III1, III5, III7, IV5, IV13, IV18, IV21 and IV23) and one unaffected male (IV19) were subjected to X-inactivation analysis as previously described ${ }^{29}$. A total of $500 \mathrm{ng}$ blood-derived DNA was digested with $10 \mathrm{U}$ of the methylation-sensitive enzyme $\mathrm{HpaII}$ at $37^{\circ} \mathrm{C}$ for $16 \mathrm{~h}$ and enzyme-inactivated at $95^{\circ} \mathrm{C}$ for $10 \mathrm{~min}$. The first exon of the androgen receptor gene $(A R)$ was amplified using a fluorescently labelled forward primer FAM-5'-CGCGAAGTGATCCAGAACCC- $3^{\prime}$ and reverse primer $5^{\prime}$-GTTGCTGTT CCTCATCCAGG- ${ }^{\prime}$ in digested and undigested DNA templates. The polymorphic CAG repeats in the $A R$ gene were used to identify parental chromosomes. Methylation differences were analysed based on peak heights in the PCR products by Genemapper version 3.7 (Applied Biosystems, Foster City, CA).

Linkage analysis. Linkage analysis for Family I was performed according to the previously described methods ${ }^{30}$. X-chromosomal microsatellite markers were PCRamplified using panel 28 of ABI Prism Linkage Mapping Sets-MD10 Version 2.5 (Applied Biosystems, Foster City, CA). An additional 21 polymorphic

microsatellite markers were used for fine mapping on chromosome Xp22 between DXS8051 and DXS1068 (Supplementary Table 3). All fluorescently labelled primers were typed on an ABI Prism 3100 genetic analyzer (Applied Biosystems, Foster City, CA) with Genemapper version 3.7. For genetic mapping, the MLINK program was used to calculate two-point linkage analysis with the following model: $\mathrm{X}$-linked recessive with high penetrance, disease and normal allele frequencies were set at 0.01 and 0.99 . Maximum logarithm of the odds (LOD) score using the deduced genotyping of unavailable members was also shown in the bracket. Linkage analysis of the X-chromosome using a panel of microsatellite markers defined a 21.6-Mb critical region from markers DXS7108 to DXS1067, with a maximum LOD score of 3.31 (Supplementary Fig. 1a and Supplementary Table 3).

Mutation analysis of candidate genes. Candidate genes PIR, TRAPPC2, PHEX, Cxorf15, AP1S2, CA5B, PDK3, PIGA, PRRG1, SMPX, were selected because of their inferred biological relevance for connective tissue functions. HCCS, MSL3, PRPS2 on the telomeric end of the critical region were also studied. Primer pairs used for amplification of entire coding regions are available on request.

Sequencing of a set of genes involved in OI and bone fragility ( $A L P L, B M P 1$, COL1A1, COL1A2, CREB3L1, CRTAP, FKBP10, IFITM5, LEPRE1, LRP5, PLOD2, PLS3, PPIB, SERPINF1, SERPINH1, SP7, TMEM38B and WNT1) was performed (CTGT, Allentown, PA). Screening of IFITM5 by PCR and Sanger sequencing was normal. Sequencing of COL1A2 revealed a single heterozygous IVS30 $+6 \mathrm{~T}>\mathrm{C}$ transition, which did not alter splicing (Supplementary Fig. 3). To determine the functionality of the change, a 260-bp fragment of genomic DNA from the OI proband, his mother, father and an affected male family member was PCR-amplified using $1.5 \mathrm{mM} \mathrm{MgCl}_{2}, 0.2 \mathrm{mM}$ dNTPs, $200 \mathrm{nM}$ forward (5'-AGCCT GTGTACTTATGCACT-3') and reverse (5'-CAGTGGCTTTAAGGAGAAAG-3') primers and $0.5 \mathrm{U}$ Taq DNA polymerase (Life Technologies, Grand Island, NY) with 35 cycles of $94^{\circ} \mathrm{C}$ for $30 \mathrm{~s}, 58^{\circ} \mathrm{C}$ for $30 \mathrm{~s}$ and $72{ }^{\circ} \mathrm{C}$ for $30 \mathrm{~s}$. To confirm that the COL1A2 IVS30 + 6T >C transition was a nonfunctional genomic SNP, normal mRNA splicing was verified by RT-PCR analysis using total RNA from control and proband fibroblasts treated with or without emetine. After removal of contaminating DNA by treatment with DNA-free DNase (Applied Biosystems, Foster City, CA), poly-A mRNA was reverse transcribed using oligo $d(T)$ and MuLV reverse transcriptase. The resulting cDNA was amplified from sequences corresponding to $\alpha 2$ (I) exons 25-32 using 1 U Platinum Taq High Fidelity (Life Technologies, Grand Island, NY) and $1.5 \mathrm{mM} \mathrm{MgSO}_{4}, 0.4 \mathrm{mM}$ dNTPs, $15 \mathrm{nmol}$ forward $\left(5^{\prime}\right.$-CATTGGATTCCCTGGACCCAAAGGCCCCAC- $\left.3^{\prime}\right)$ and reverse (5'-GGAGTCCACTAGGACCAGATGGACCAGCAG- $\left.3^{\prime}\right)$ primers, and 35 cycles of $94^{\circ} \mathrm{C}$ for $1 \mathrm{~min}, 67^{\circ} \mathrm{C}$ for $20 \mathrm{~s}$ and $72{ }^{\circ} \mathrm{C}$ for $45 \mathrm{~s}$. PCR products were electrophoresed on $6 \%$ polyacrylamide and visualized by ethidium bromide staining.

Comparative Genomic Hybridization. CGH was performed on test DNA (affected male, IV17) and reference DNA (unaffected male, IV10) for the determination of copy number variants (Macrogen Inc., Seoul, Korea). DNAs were independently labelled with fluorescent dyes, co-hybridized to a NimbleGen Human CGH 385K chromosome X Tiling array, and scanned using a $2-\mu \mathrm{m}$ scanner. $\log 2$-ratio values of the probe signal intensities $(\mathrm{Cy} 3 / \mathrm{Cy} 5)$ were calculated and plotted versus genomic position using Roche NimbleGen NimbleScan software. Data are displayed in Roche NimbleGen SignalMap software. CGH did not show any imbalances in the critical region.

Next-generation sequencing. We performed targeted resequencing by NGS of the 18.6-MB linkage region between DXS1224 and DXS1067 interval on affected male (IV17) and unaffected male (IV10) genomic DNAs (Macrogen Inc., Seoul, Korea). DNA was captured on a customized NimbleGen 2.1 array (Roche NimbleGen, Madison, WI) with a capturing capacity of $33 \mathrm{Mb}$. The targeted region corresponded to positions $13,235,460$ to $31,798,001$ bp on Chromosome Xp22 according to the UCSC hg19 Assembly. Capture efficiency varied across the target, with a mean read depth of $121.1 \mathrm{X}$. The captured library was subsequently sequenced using the Illumina platform Genome Analyzer II X (GAIIX) in a single-end 76 bp configuration. Sequence reads were mapped against UCSC hg19 using BWA software (http://bio-bwa.sourceforge.net/). The SNPs and Indels were detected by SAMTOOLS (http://samtools.sourceforge.net/) and annotated by SIFT (http://sift.jcvi.org/) (Supplementary Table 4). We found 25 variants in coding sequences. After excluding known SNPs in dbSNP Build 135, 1000 Genomes and HAPMAP, and variants present in the unaffected relative, two candidates remained: c.1376A > G (p.N459S) in MBTPS2 and c.532G > A (p.V178M) in FAM48B1.

Mutation confirmation and segregation studies. PCR amplification and Sanger sequencing were performed to confirm the MBTPS2 mutation in the proband and his parents. Primer pairs and PCR conditions for the amplification of the coding exons of MBTPS2 including the intron-exon boundaries are available on request. Restriction enzyme digestion with BsgI was used for cosegregation study and normal control screening. The identified mutation was analysed with ClustalX for conservation analysis. Alamut, Polyphen2, SIFT, Panther and align GVGD were used for prediction of mutation function.

Molecular genetic investigations of Family II. Sequencing of osteogenesis imperfecta candidate genes. Exclusion of disease-causing mutations segregating with the phenotype was performed for ALPL, BMP1, COL1A1, COL1A2, CREB3L1, CRTAP, FKBP10, IFITM5, LEPRE1, LRP5, PLOD2, PLS3, PPIB, SERPINF1, SERPINH1, SP7, TMEM $38 B$ and WNT1 (CTGT, Allentown, PA). A heterozygous c.4018G > A (p.G1340S) in COL1A1, reported as rs147936946 in the dbSNP database was found in P1/II and his healthy father. This variant was absent in the affected maternal uncle (P2/II), as well as in the proband's mother (Supplementary Fig. 3c).

Genome-wide linkage analysis. For the five living members of Family II (Supplementary Fig. 1) a genome-wide linkage scan was performed using the HumanCytoSNP-12v2 BeadChip (Illumina, San Diego, CA) according to the supplier's instructions. Raw SNP call data were processed with the Genotyping Analysis Module in GenomeStudio 1.6.3 (Illumina, San Diego, CA). Homozygosity mapping was performed with the Allegro program, under the assumption of a recessive mode of inheritance in absence of consanguinity loops.

$X$-inactivation assay. X-inactivation analysis was performed on gDNA from the mother of P1/II according to Beever $e^{2} a^{31}$. PCR products were analysed on an ABI 
3130 Capillary DNA Sequencer (Applied Biosystems) and the methylation status was analysed by Genemapper version 4.0 (Applied Biosystems) based on the peak heights of the PCR products.

Exome enrichment and high-throughput sequencing. Genomic DNA was extracted from whole blood using the MagNA Pure Compact System (Roche Life Science, Indianapolis, IN, USA). DNA library preparation was performed with the TruSeq DNA Sample Preparation Kit (Illumina, San Diego, CA) according to the manual instructions. Enrichment of X-chromosomal genes was done by using a custom designed NimbleGen Sequence Capture Microarray (solid array with 6962 targets on the $\mathrm{X}$ chromosome or $1.4 \mathrm{Mb}$ ). The captured library was sequenced on a MiSeq sequencer (Illumina, v1) with $2 \times 150$ cycles of paired-end sequencing.

Data analysis. Raw data processing, sequence read alignment from FASTQ to BAM format and variant calling to generate VCF files were performed with the MiSeqReporter Software (Illumina, San Diego, CA). Aligned BAM files with removed duplicated reads were further technically analysed using the SeqMonk program version v0.25.0 (http://www.bioinformatics.babraham.ac.uk/projects/ seqmonk). Variant filtering was performed by GeneTalk (https://www.gene-talk.de/).

Mutation confirmation and segregation study. Mutation screening of MBTPS2 and segregation analysis of the mutation within the family was performed by fluorescent bidirectional sequencing of genomic amplified PCR products on an ABI 3100 automated sequence detection system (Applied Biosystems, Foster City, CA, USA). PCR conditions and primer sequences are available from the authors on request.

Bioinformatics analysis aimed to predict pathogenicity of the identified L505F variant was performed with Provean, Panther, MutationTaster and PolyPhen2. Conservation analysis of the protein variant was performed by multiple sequence alignment of MBTPS2 with ClustalX, ClustalW and BOXSHADE programs (Fig. 2e).

Urinary pyridinoline analysis. Total urinary pyridinoline crosslinks, LP and HP, were measured in the affected individuals and obligate carriers of Family I and Family II, as well as in an MBTPS2 R429H IFAP patient and unaffected carrier (Supplementary Table 2) by HPLC as described ${ }^{32}$.

Luciferase assay. CHO-M19 cells, a deleted Mbtps2 orthologous cell line, were used for transfection of reporter constructs. Cells were maintained in Ham's F-12 medium supplemented with $10 \%$ fetal bovine serum at $37^{\circ} \mathrm{C}$ with $5 \% \mathrm{CO}_{2}$. We performed luciferase assays by transient transfection of CHO-M19 cells either with p5xATF6 or pSRE reporters as previously described ${ }^{5,7}$. The p5xATF6 and pSRE reporters contain either an ER-stress response element or a sterol regulatory element, respectively, two elements that are known targets of S2P. These assays are an indirect measurement of the transcriptional activity of S2P cleavage products on these two elements. For the p5xATF6 reporter, cells were set up at a density of $1.5 \times 10^{5}$ cells per well in triplicate in 12-well culture plates. Cells were transfected with $1 \mu \mathrm{g}$ of expression plasmid containing normal, mutant (N459S, R429H and L505F) MBTPS2 or no insert, together with $800 \mathrm{ng}$ of p5xATF6-GL3 and $50 \mathrm{ng}$ of pRL-SV40 as a transfection control. The total amount of DNA was adjusted to $2 \mu \mathrm{g}$ per dish by addition of pdEYFP-Clamp. At $24 \mathrm{~h}$ post-transfection, cells were treated with $2 \mu \mathrm{g} \mathrm{ml}^{-1}$ of tunicamycin dissolved in DMSO to induce stress. Untreated cells received $0.2 \%$ DMSO. After incubation for $24 \mathrm{~h}$, the firefly and renilla luciferase activities were measured using a dual luciferase assay system according to the manufacturer's protocol (E1910 Promega kit, Madison, WI). For the pSRE-reporter, cells were set up at a density of $1.5 \times 10^{5}$ cells per well in triplicate in a 12 -well plate. On day 2 , the cells were switched to medium containing 1:1 Ham's F12:DMEM, 5\% lipoprotein-deficient serum and $20 \mathrm{mM}$ sodium oleate for $7 \mathrm{~h}$. The transfected plasmids were either wild-type S2P or one of the three mutants (p.N459S, p.R429H, p.H171F) and the pSRE reporter. Cells were then switched to medium containing 1:1 Ham's F12:DMEM, 5\% lipoproteindeficient serum, $50 \mathrm{mM}$ compactin and $50 \mathrm{mM}$ mevalonate supplemented with sterol ( $10 \mathrm{mg} \mathrm{ml}^{-1}$ cholesterol, $1 \mathrm{mg} \mathrm{ml}^{-1}$ 25-hydroxycholesterol). Untreated cells were treated with $0.2 \%$ ethanol $(0.2 \% \mathrm{w} / \mathrm{v})$. The firefly and renilla luciferase activities were measured after $16 \mathrm{~h}$ of incubation. The ratio between firefly luciferase and renilla luciferase luminescence from different constructs and control was analysed by one-way analysis of variance (ANOVA). Specific pairwise comparison between luciferase luminescence in each group used the least significant difference test. One-way ANOVA and least significant difference were included in SPSS software version 22.

Western blot analysis. Cell lysates from primary fibroblasts and osteoblasts were collected in RIPA buffer supplemented with a protease inhibitor cocktail (Sigma-Aldrich, St Louis, MO), and used for western blotting of MBTPS2 (Cell Signaling Technology, Danvers, MA), OASIS (R\&D Systems, Minneapolis, MN) and PLOD1/LH1 (Abgent Inc., San Diego, CA) proteins. Cells were grown to confluence and treated with tunicamycin $\left(2 \mu \mathrm{g} \mathrm{ml}^{-1}\right)$, thapsigargin $(1 \mathrm{mM})$ and/or ALLN $\left(25 \mu \mathrm{g} \mathrm{ml}^{-1}\right)$ for $2 \mathrm{~h}$ before harvest. Protein lysate $(50 \mu \mathrm{g})$ was loaded per lane on a $4-20 \%$ (MBTPS2, PLOD1) or $10 \%$ (OASIS) Tris-Glycine Ready gel (Bio-Rad, Hercules, CA), blotted onto $0.2 \mu \mathrm{m}$ nitrocellulose and blocked with Odyssey blocking buffer (MBTPS2) or 5\% BSA plus $1 \times$ casein (OASIS, PLOD1) before probing with antibody overnight. Blots were washed, incubated with secondary IR-conjugated antibodies for $1 \mathrm{~h}$, washed and visualized on a LI-COR Odyssey infrared imager (LI-COR, Lincoln, NE).
Collagen biochemical studies. Dermal fibroblast cultures were established from skin punch biopsies and grown in Dulbecco's Modified Eagle Medium (DMEM) containing $10 \%$ fetal bovine serum (FBS), $100 \mathrm{U} \mathrm{ml}^{-1}$ penicillin and $100 \mu \mathrm{g} \mathrm{ml}^{-1}$ streptomycin.

For type I collagen biochemical studies, normal control and proband primary fibroblast and osteoblast (see below) cultures were labelled for $16 \mathrm{~h}$ in serum-free DMEM containing $437.5 \mu \mathrm{Ci} \mathrm{ml}^{-1} \mathrm{~L}-\left[2,3,4,5-{ }^{3} \mathrm{H}\right]$-proline. Procollagens were precipitated with ammonium sulfate, pepsin digested and electrophoresed on $6 \%$ SDS-urea polyacrylamide gels under nonreducing conditions ${ }^{33}$. Procollagen secretion by control and proband fibroblasts (Fig. 5b) was determined in 24-h conditioned media using the Sircol collagen assay (Biocolor Ltd, Carrickfergus, UK) according to the manufacturer's specifications. Collagen protease sensitivity was analysed as previously described ${ }^{34}$. For protease sensitivity assays, type I collagen from the medium of cultured proband fibroblasts labelled overnight with $437.5 \mu \mathrm{Ci} \mathrm{ml}^{-1} \mathrm{~L}-\left[2,3,4,5{ }^{3} \mathrm{H}\right]$-proline was digested for 1,3 and $5 \mathrm{~min}$ with trypsin and chymotrypsin (100 and $250 \mathrm{mg} \mathrm{ml}^{-1}$ final concentration, respectively) at $37^{\circ} \mathrm{C}$ without prior cooling. Digests were stopped with $1 \mathrm{mg} \mathrm{ml}^{-1}$ soybean trypsin inhibitor and analysed by SDS-urea PAGE.

Differential scanning calorimetry (DSC) was performed in buffer containing $0.2 \mathrm{M}$ sodium phosphate and $0.5 \mathrm{M}$ glycerol at $\mathrm{pH} 7.4$, from 10 to $55^{\circ} \mathrm{C}$ in a Nano III DSC instrument (Calorimetry Sciences Corporation, Lindon, UT), as previously described ${ }^{35}$. Amino-acid composition was analysed by high pressure liquid chromatography (AIBiotech, Richmond, VA).

Collagen extracellular matrix deposition was analysed as previously described ${ }^{36}$. In brief, post-confluent proband and normal control fibroblasts were cultured for 2 weeks in DMEM containing $10 \%$ FBS with $100 \mu \mathrm{g} \mathrm{ml}^{-1}$ ascorbic acid. After 14 days, fibroblasts were labelled $24 \mathrm{~h}$ with $406.25 \mu \mathrm{Ci} \mathrm{ml}{ }^{-1} \mathrm{~L}-\left[2,3,4,5-{ }^{3} \mathrm{H}\right]$-proline and the media and extracellular matrix was harvested. Collagens were sequentially extracted from the matrix with $150 \mathrm{mM} \mathrm{NaCl}, 0.5 \mathrm{~N}$ acetic acid and then $50 \mu \mathrm{g} \mathrm{ml}^{-1}$ pepsin.

Expression studies. Total fibroblast RNA was extracted from primary fibroblasts using TriReagent (Molecular Research Center, Cincinnati, OH), followed by DNase treatment and RNA integrity verification on an Agilent 2100 Bioanalyzer (Agilent, Santa Clara, CA). Complementary DNA was reverse transcribed from $5 \mu \mathrm{g}$ RNA using a High-Capacity cDNA Archive Kit (Life Technologies Corporation, Carlsbad, CA). Taqman Gene Expression Assays were used to determine the transcript level of MBTPS2 (Hs00210639_m1), COL1A1 (Hs00164004_m1), COL1A2 (Hs00164099_m1), PLOD1 (Hs00609368_m1), CREB3L1

(Hs00223565_m1), ALPL (Hs01029144_m1), MATN1 (Hs00159075_m1) and SMAD4 (Hs00929647_m1) (Life Technologies Corporation, Carlsbad, CA). Relative expression of each gene of interest was compared with the expression of GAPDH (Hs99999905_m1) and normalized to normal control primary fibroblasts.

Osteoblast studies. Osteoblast primary cultures were established from surgical bone chips and cultured in $\alpha$ MEM (Life Technologies, Grand Island, NY) supplemented with $10 \% \mathrm{FBS}$, penicillin and streptomycin at $37^{\circ} \mathrm{C}$ and $8 \% \mathrm{CO}_{2}$ (ref. 37). Only passages 1 and 2 were used for experiments. For differentiation, osteoblast cultures were grown to confluence and treated with differentiation medium ( $\alpha$ MEM containing $10 \% \mathrm{FBS}, 25 \mu \mathrm{g} \mathrm{ml}{ }^{-1} \mathrm{~L}$-ascorbic acid, dexamethasone $\left(10^{-8} \mathrm{M}\right)$, and $2.5 \mathrm{mM}$ 2-glycerophosphate for 3 weeks.

Mass spectral analysis of bone tissue collagen. Demineralized bone was digested with bacterial collagenase and the resulting collagen-derived peptides were separated by reverse-phase HPLC, as previously described ${ }^{32}$. Electrospray MS was performed on in-gel trypsin digests and individual HPLC column fractions using an LTQ XL ion-trap mass spectrometer equipped with in-line liquid chromatography (Thermo Scientific, Waltham, MA) using a C4 5- $\mu \mathrm{m}$ capillary column $(300 \times 150 \mathrm{~mm}$; Higgins Analytical RS-15M3-W045) eluted at $4.5 \mu \mathrm{lmin}-1$. The LC mobile phase consisted of buffer A $(0.1 \%$ formic acid in MilliQ water) and buffer B (0.1\% formic acid in 3:1 acetonitrile:n-propanol v/v). The LC sample stream was introduced into the mass spectrometer by electrospray ionization with a spray voltage of $4 \mathrm{kV}$. Proteome Discoverer search software (Thermo Scientific) was used for peptide identification using the NCBI protein database. Proline and lysine modifications were examined manually by scrolling or averaging the full scan over several minutes so that all of the post-translational variations of a given peptide appeared together in the full scan.

Study oversight. This study was conducted according to the Declaration of Helsinki for Human Rights and approved by Swiss Ethics and by the Institutional Review Board of the Faculty of Medicine, Chulalongkorn University. Written informed consent was obtained from the patients or their parents.

Data availability. The data that support the findings of this study are available from the corresponding author on request. 


\section{References}

1. Forlino, A. \& Marini, J. C. Osteogenesis imperfecta. Lancet 387, 1657-1671 (2016).

2. Mendoza-Londono, R. et al. Recessive osteogenesis imperfecta caused by missense mutations in SPARC. Am. J. Hum. Genet. 96, 979-985 (2015).

3. Lal, M. \& Caplan, M. Regulated intramembrane proteolysis: signaling pathways and biological functions. Physiology (Bethesda) 26, 34-44 (2011).

4. Murakami, T. et al. Cleavage of the membrane-bound transcription factor OASIS in response to endoplasmic reticulum stress. J. Neurochem. 96, 1090-1100 (2006).

5. Ye, J. et al. ER stress induces cleavage of membrane-bound ATF6 by the same proteases that process SREBPs. Mol. Cell 6, 1355-1364 (2000).

6. Wang, X., Sato, R., Brown, M. S., Hua, X. \& Goldstein, J. L. SREBP-1, a membrane-bound transcription factor released by sterol-regulated proteolysis. Cell 77, 53-62 (1994).

7. Oeffner, F. et al. IFAP syndrome is caused by deficiency in MBTPS2, an intramembrane zinc metalloprotease essential for cholesterol homeostasis and ER stress response. Am. J. Hum. Genet. 84, 459-467 (2009).

8. Naiki, M. et al. MBTPS2 mutation causes BRESEK/BRESHECK syndrome. Am. J. Med. Genet. A 158A, 97-102 (2011).

9. Aten, E. et al. Keratosis Follicularis Spinulosa Decalvans is caused by mutations in MBTPS2. Hum. Mutat. 31, 1125-1133 (2010).

10. Rudner, D. Z., Fawcett, P. \& Losick, R. A family of membrane-embedded metalloproteases involved in regulated proteolysis of membrane-associated transcription factors. Proc. Natl Acad. Sci. USA 96, 14765-14770 (1999).

11. Lindert, U. et al. Urinary pyridinoline cross-links as biomarkers of osteogenesis imperfecta. Orphanet. J. Rare. Dis. 10, 104 (2015).

12. Cabral, W. A. et al. Abnormal type I collagen post-translational modification and crosslinking in a cyclophilin B KO mouse model of recessive osteogenesis imperfecta. PLoS Genet. 10, e1004465 (2014).

13. Murakami, T. et al. Signalling mediated by the endoplasmic reticulum stress transducer OASIS is involved in bone formation. Nat. Cell. Biol. 11, 1205-1211 (2009).

14. Chen, Q., Lee, C. E., Denard, B. \& Ye, J. Sustained induction of collagen synthesis by TGF-beta requires regulated intramembrane proteolysis of CREB3L1. PLOS ONE 9, e108528 (2014).

15. Vellanki, R. N. et al. OASIS/CREB3L1 induces expression of genes involved in extracellular matrix production but not classical endoplasmic reticulum stress response genes in pancreatic beta-cells. Endocrinology 151, 4146-4157 (2010).

16. Ishikawa, Y. et al. Mutation in cyclophilin B that causes hyperelastosis cutis in American Quarter Horse does not affect peptidylprolyl cis-trans isomerase activity but shows altered cyclophilin B-protein interactions and affects collagen folding. J. Biol. Chem. 287, 22253-22265 (2012).

17. van Dijk, F. S. et al. PLS3 mutations in X-linked osteoporosis with fractures. N. Engl. J. Med. 369, 1529-1536 (2013).

18. Rawson, R. B. The site-2 protease. Biochim. Biophys. Acta 1828, 2801-2807 (2013).

19. Haghighi, A. et al. A missense mutation in the MBTPS2 gene underlies the X-linked form of Olmsted Syndrome. J. Invest. Dermatol. 133, 571-573 (2013).

20. Bornholdt, D. et al. Genotype-phenotype correlations emerging from the identification of missense mutations in MBTPS2. Hum. Mutat. 34, 587-594 (2013).

21. Rawson, R. B. Control of lipid metabolism by regulated intramembrane proteolysis of sterol regulatory element binding proteins (SREBPs). Biochem. Soc. Symp. 70, 221-231 (2003).

22. Schlombs, K., Wagner, T. \& Scheel, J. Site-1 protease is required for cartilage development in zebrafish. Proc. Natl Acad. Sci. USA 100, 14024-14029 (2003).

23. Patra, D. et al. Site-1 protease is essential for endochondral bone formation in mice. J. Cell Biol. 179, 687-700 (2007).

24. Patra, D., DeLassus, E., Liang, G. \& Sandell, L. J. Cartilage-specific ablation of site-1 protease in mice results in the endoplasmic reticulum entrapment of type IIb procollagen and down-regulation of cholesterol and lipid homeostasis. PLoS ONE 9, e105674 (2014)

25. Symoens, S. et al. Deficiency for the ER-stress transducer OASIS causes severe recessive osteogenesis imperfecta in humans. Orphanet J. Rare Dis. 8, 154 (2013).

26. Eyre, D. R. \& Weis, M. A. Bone collagen: new clues to its mineralization mechanism from recessive osteogenesis imperfecta. Calcif. Tissue Int. 93, 338-347 (2013)

27. Kondo, S., Saito, A., Asada, R., Kanemoto, S. \& Imaizumi, K. Physiological unfolded protein response regulated by OASIS family members, transmembrane bZIP transcription factors. IUBMB Life 63, 233-239 (2011).

28. Pietrzak, A. et al. IFAP syndrome with severe cutaneous, neurologic and skeletal manifestations due to a novel MBTPS2 mutation in a Polish patient. Eur. J. Dermatol. 22, 467-472 (2012).
29. Leoyklang, P., Suphapeetiporn, K., Wananukul, S. \& Shotelersuk, V. Three novel mutations in the PORCN gene underlying focal dermal hypoplasia. Clin. Genet. 73, 373-379 (2008).

30. Yeetong, P. et al. A newly identified locus for benign adult familial myoclonic epilepsy on chromosome 3q26.32-3q28. Eur. J. Hum. Genet. 21, 225-228 (2013).

31. Beever, C. L., Penaherrera, M. S., Langlois, S. \& Robinson, W. R. X chromosome inactivation patterns in Russell-Silver syndrome patients and their mothers. Am. J. Med. Genet. A 123A, 231-235 (2003).

32. Lindert, U. et al. Molecular consequences of the SERPINH1/HSP47 mutation in the Dachshund Natural Model of osteogenesis imperfecta. J. Biol. Chem. 290, 17679-17689 (2015).

33. Bonadio, J., Holbrook, K. A., Gelinas, R. E., Jacob, J. \& Byers, P. H. Altered triple helical structure of type I procollagen in lethal perinatal osteogenesis imperfecta. J. Biol. Chem. 260, 1734-1742 (1985).

34. Christiansen, H. E. et al. Homozygosity for a missense mutation in SERPINH1, which encodes the collagen chaperone protein HSP47, results in severe recessive osteogenesis imperfecta. Am. J. Hum. Genet. 86, 389-398 (2010).

35. Cabral, W. A. et al. Type I collagen triplet duplication mutation in lethal osteogenesis imperfecta shifts register of alpha chains throughout the helix and disrupts incorporation of mutant helices into fibrils and extracellular matrix. J. Biol. Chem. 278, 10006-10012 (2003).

36. Bateman, J. F. \& Golub, S. B. Deposition and selective degradation of structurally-abnormal type I collagen in a collagen matrix produced by osteogenesis imperfecta fibroblasts in vitro. Matrix Biol. 14, 251-262 (1994).

37. Robey, P. G. \& Termine, J. D. Human bone cells in vitro. Calcif. Tissue Int. 37, 453-460 (1985).

\section{Acknowledgements}

We thank the patients and their families for participating in this study and supporting our work. Osteoblast cultures were kindly established by Professor Thomas Braulke, University Medical Center Hamburg-Eppendorf, Children's Hospital, Hamburg, Germany. CHO-M19, EYFP-MBTPS2, pSRE-GL4.23 and pRL-SV40 constructs were kindly provided by Professor Karl-Heinz Grzeschik, Department of Human Genetics, Philipps-Universität, Marburg, Germany. IFAP fibroblast cultures were generously provided by Dr Cynthia J. Tifft, Pediatric Undiagnosed Diseases Program, NHGRI, NIH. KFSD fibroblast cultures were kindly provided by Dr Emmelien Aten, Department of Human and Clinical Genetics, LUMC, Leiden, The Netherlands. The p5xATF6-GL3 was kindly provided by Ron Prywes of Department of Biological Sciences, Columbia University, New York, USA. We thank Angelika Schwarze and Anke Jeschke for expert technical assistance, and Professor Marius Kraenzlin for urinary pyridinoline measurement. This study was supported by the Thailand Research Fund (RTA56800003 and TRG5480013) and the Chulalongkorn Academic Advancement into its 2nd century project to V.S., by the Swiss National Research Foundation (SNF Grant No. 310030_138288) to C.G. and M.R., by NIH grants AR037318 (NIAMS) and HD070394 (NICHD) to D.R.E., and by NICHD Intramural Funds to J.C.M. and S.L. The illustration in Fig. 6c was generated by Yumiko Shepherd, Unit of Computer Support Services, NICHD.

\section{Author contributions}

S.A. and P.Y. performed X-inactivation studies, linkage analysis, analysed NGS and CGH data and performed functional experiments of the identified mutation for Family I. Also for Family I, C.S. prepared samples, and did PCR analyses of collagen and gene sequencing; S.T. conducted reporter assays and functional studies. For Family II, I.K. ascertained the family and elaborated the clinical data. U.L. performed collagen biochemical analyses, expression and functional studies of MBTPS2. A.J. and B.K. performed linkage analyses, and K.L. and B.R. did X-inactivation studies and NGS. E.M. conducted DSC under the supervision of S.L. M.W. and D.E. conducted mass spectroscopy analyses of P1/II bone sample. A.M.B. produced immunoblots of MBTPS2, PLOD1 and OASIS cleavage. W.A.C. performed collagen biochemical analyses, expression quantitation of MBTPS2 and PLOD1 and studies of cultured osteoblasts. Patient samples were collected by K.S., V.S. (Family I) and I.K., M.R. and C.G. (Family II). J.C.M., K.S., V.S., M.R. and C.G. designed and supervised the study. J.C.M. drafted the manuscript with significant input from K.S., V.S., W.A.C., U.L. and C.G. All authors read and approved the final manuscript.

\section{Additional information}

Supplementary Information accompanies this paper at http://www.nature.com/ naturecommunications

Competing financial interests: The authors declare no competing financial interests.

Reprints and permission information is available online at http://npg.nature.com/ reprintsandpermissions/ 
How to cite this article: Lindert, U. et al. MBTPS2 mutations cause defective regulated intramembrane proteolysis in X-linked osteogenesis imperfecta. Nat. Commun. 7:11920 doi: 10.1038/ncomms11920 (2016).

(c) (1) This work is licensed under a Creative Commons Attribution 4.0 International License. The images or other third party material in this article are included in the article's Creative Commons license, unless indicated otherwise in the credit line; if the material is not included under the Creative Commons license, users will need to obtain permission from the license holder to reproduce the material. To view a copy of this license, visit http://creativecommons.org/licenses/by/4.0/

(C) The Author(s) 2016 